\title{
The physical nature of ethidium and proflavine interactions with nucleic acid bases in the intercalation plane Supporting Information
}

\author{
Karol M. Langner ${ }^{1}$, Pawel Kedzierski ${ }^{1}$ \\ W. Andrzej Sokalski ${ }^{1}$, Jerzy Leszczynski ${ }^{2}$ \\ ${ }^{1}$ Wroclaw University of Technology, Poland \\ ${ }^{2}$ Jackson State University, MS, USA
}

\section{S.1 Dependence of interaction energy components on the basis set}

Components of the $\Delta E_{\mathrm{MP} 2}$ interaction between the ethidium chromophore and one uracyl base in the $\mathrm{Eth}^{(+1)} \mathrm{L}_{-}$ $\mathrm{UA} / \mathrm{AU}$ complex, obtained with various basis sets, are shown. The basis 6-31G(1d,1p) was used throughout the study. Note the strong dependence of the electron correlation term, $\Delta E_{\mathrm{corr}}^{(R)}$. All values are in $\mathrm{kcal} / \mathrm{mol}$.

\begin{tabular}{l|ccc|cc|cc|cc} 
& $\Delta E_{\text {el,mtp }}^{(1)}$ & $\Delta E_{\text {el,pen }}^{(1)}$ & $\Delta E_{\text {el }}^{(1)}$ & $\Delta E_{\text {ex }}^{(1)}$ & $\Delta E^{(1)}$ & $\Delta E_{\text {del }}^{(R)}$ & $\Delta E_{\mathrm{SCF}}$ & $\Delta E_{\text {corr }}^{(R)}$ & $\Delta E_{\mathrm{MP} 2}$ \\
\hline 6-31G(1d,1p) & -3.88 & -1.56 & -5.44 & 4.87 & -0.57 & -0.78 & -1.35 & -4.19 & -5.54 \\
6-31G(2d,2p) & -2.80 & -2.50 & -5.29 & 4.86 & -0.43 & -0.86 & -1.30 & -5.85 & -7.15 \\
6-31G(3d,3p) & - & - & -5.28 & 4.79 & -0.47 & -1.04 & -1.52 & -7.18 & -8.70 \\
DZV(1d,1p) & -2.96 & -2.43 & -5.39 & 4.79 & -0.61 & -0.97 & -1.58 & -4.92 & -6.50 \\
DZV(2d,2p) & -2.95 & -2.31 & -5.26 & 4.81 & -0.45 & -1.00 & -1.45 & -6.36 & -7.81 \\
TZV(1d,1p) & -3.56 & -1.85 & -5.41 & 4.83 & -0.57 & -1.04 & -1.61 & -5.56 & -7.18 \\
TZV(2d,2p) & -3.14 & -2.11 & -5.25 & 4.85 & -0.40 & -1.07 & -1.47 & -6.72 & -8.19
\end{tabular}

1 Convergence of $\Delta \varepsilon_{C A M M}$ multipole interactions for the crystallographic structures

The numerical values of the interactions shown in Fig.4 of the manuscript are presented here. Adsitionally, CAMM interactions in the neutral proflavine complex and interactions due to molecular multipole expansions in the $\mathrm{Eth}^{(+1)}$-UA/AU complex are included. All values are in $\mathrm{kcal} / \mathrm{mol}$.

\begin{tabular}{c|cc|c|cc} 
& \multicolumn{2}{|c|}{$\mathrm{Eth}^{(+1)}-\mathrm{UA} / \mathrm{AU}$} & $\mathrm{Eth}^{(+1)}-\mathrm{GC} / \mathrm{CG}$ & $\mathrm{PF}^{(+1)}-\mathrm{AU} / \mathrm{CG}$ & $\mathrm{PF}^{(0)}-\mathrm{AU} / \mathrm{CG}$ \\
& $\mathrm{CAMM}$ & molecular & CAMM & CAMM & CAMM \\
\hline$\Delta \varepsilon_{C A M M}^{0}$ & -11.52 & 0.00 & -13.42 & -13.68 & 2.32 \\
$\Delta \varepsilon_{C A M M}^{I}$ & -10.45 & -30.47 & -12.19 & -13.59 & -2.48 \\
$\Delta \varepsilon_{C A M M}^{I I}$ & -9.04 & -34.1 & -10.52 & -12.82 & 4.73 \\
$\Delta \varepsilon_{C A M M}^{I I I}$ & -10.71 & 21.80 & -13.75 & -14.95 & 1.53 \\
$\Delta \varepsilon_{C A M M}^{I V}$ & -12.92 & -53.81 & -15.63 & -16.48 & 0.07 \\
$\Delta \varepsilon_{C A M M}^{V}$ & -10.51 & 53.49 & -13.16 & -14.46 & 2.51 \\
$\Delta \varepsilon_{C A M M}^{V I}$ & -10.12 & 633.02 & -12.56 & -13.99 & 3.03 \\
$\Delta \varepsilon_{C A M M}^{V I}$ & -10.58 & -649.30 & -13.05 & -14.49 & -1.98 \\
$\Delta \varepsilon_{C A M M}^{V I I}$ & -10.54 & -892.21 & -13.07 & -14.52 & 4.01 \\
$\Delta \varepsilon_{C A M M}^{I X}$ & -10.48 & 2162.98 & -13.04 & -14.44 & 0.71
\end{tabular}


2 Converged $\left(\triangle \varepsilon_{C A M M}^{I X}\right)$ multipole interaction surfaces in the intercalation plane.

Surface maps, analogical to the one shown in Fig.7 of the manuscript, are presented for the other studied systems.

2.1 Ethidium $^{(+1)}$-GC-CG complex

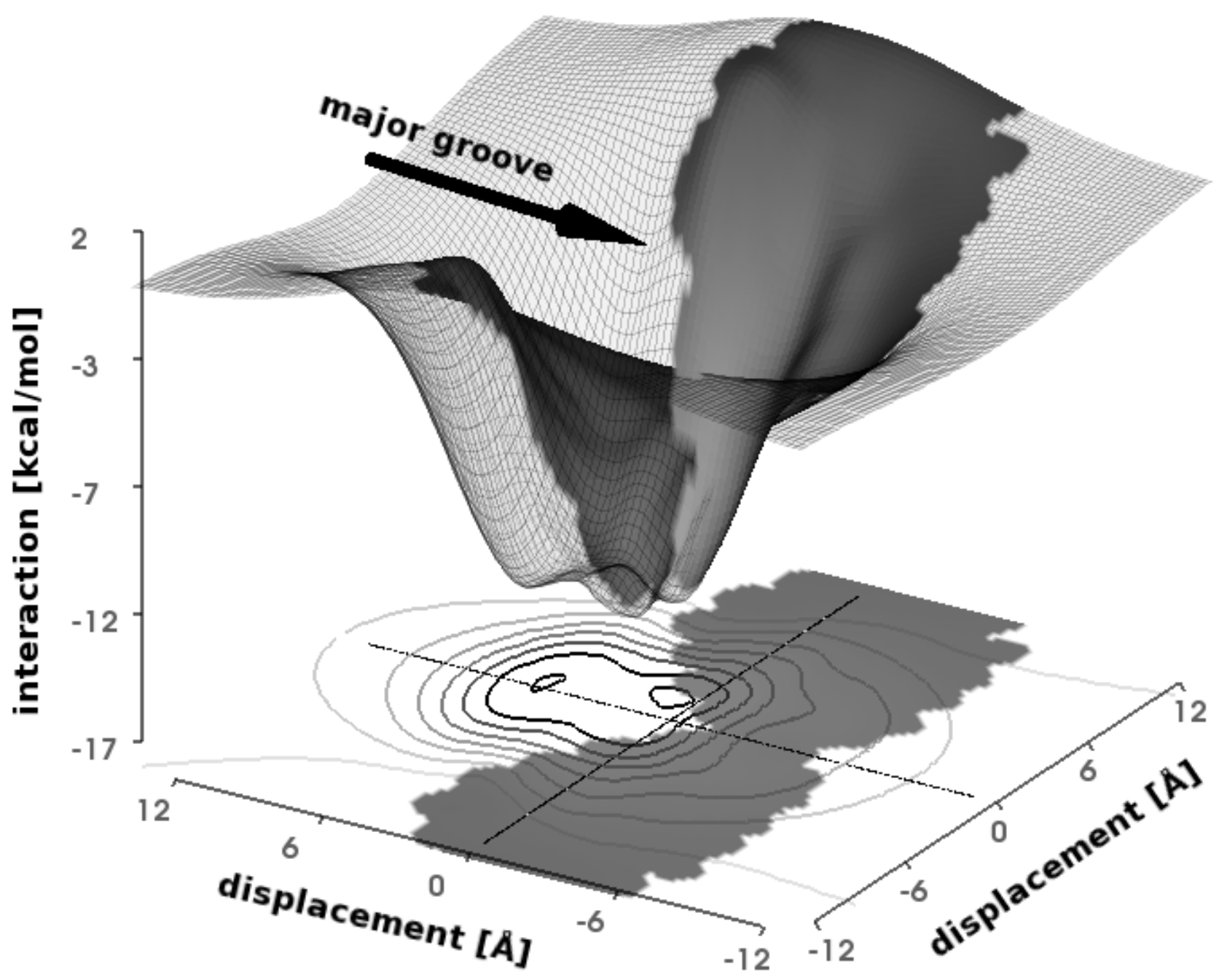


2.2 Proflavine $^{(+1)}-\mathrm{AU} / \mathrm{CG}$ complex

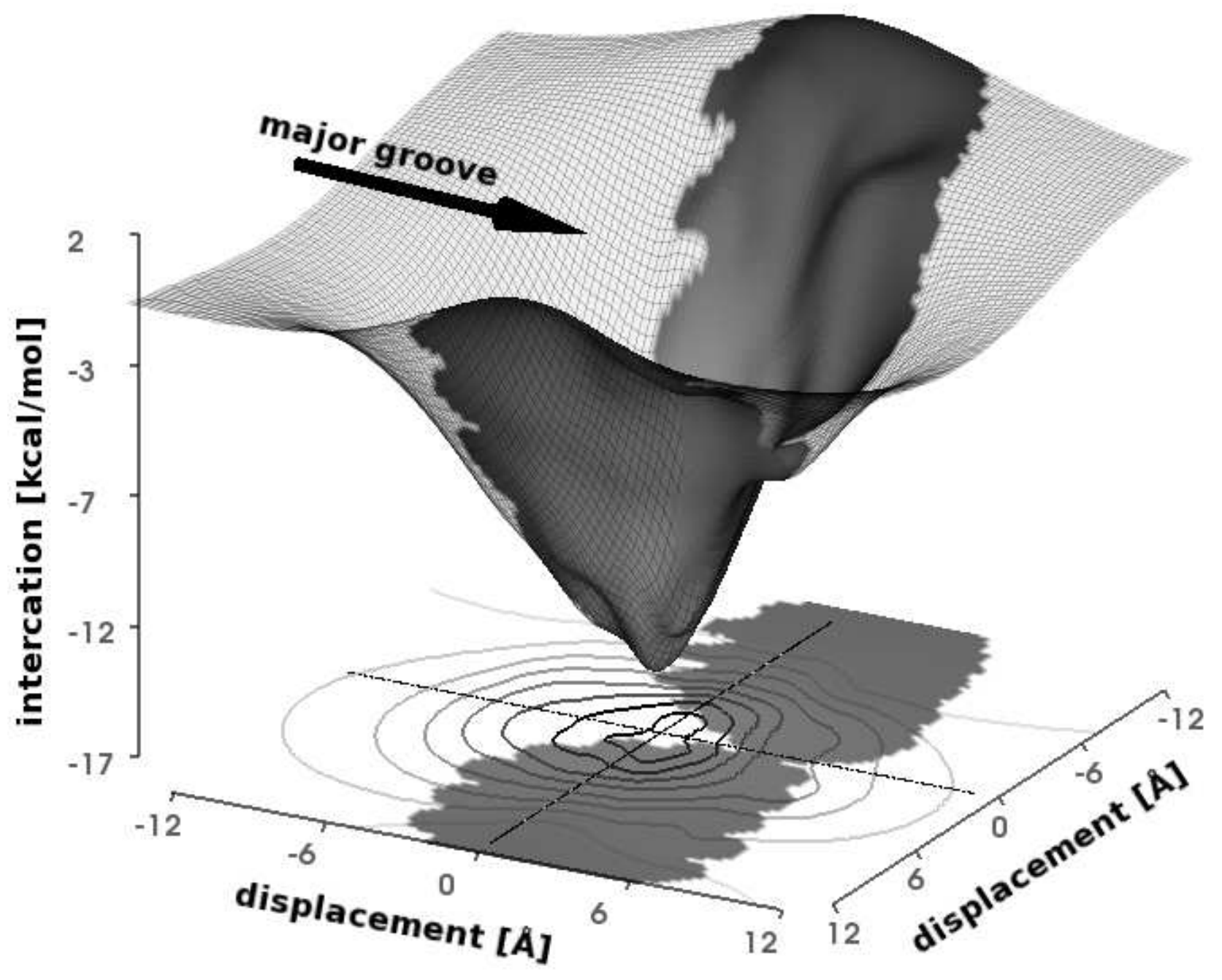


2.3 Proflavine ${ }^{(0)}-$ AU/CG complex

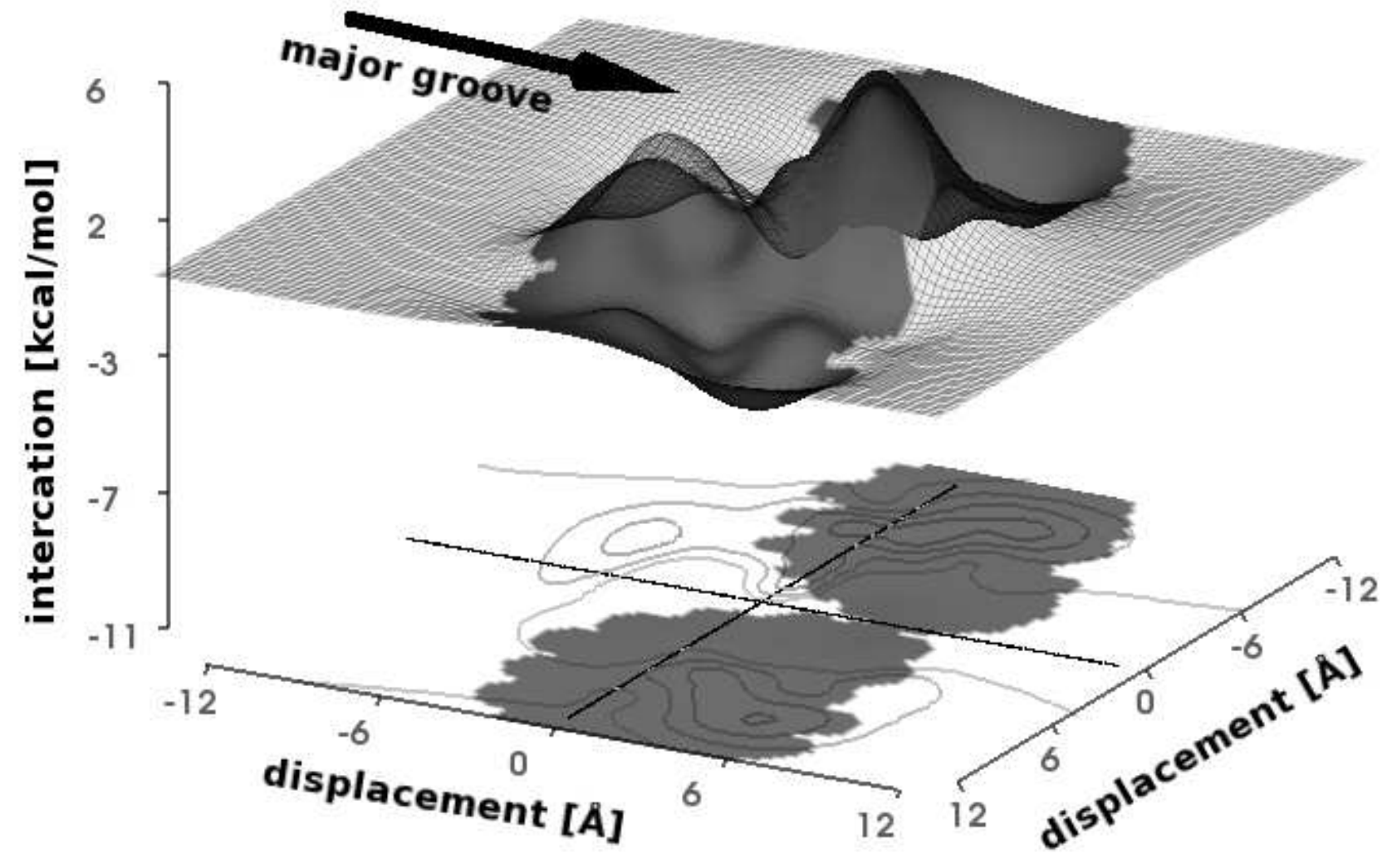




\section{Convergence of $\Delta \varepsilon_{C A M M}$ multipole interaction surfaces in the intercalation plane}

To illustrate the convergence of the CAMM interaction on the whole intercalation plane (Fig.4 in the manuscript shows the convergence at the crystallographic alignment of the chromophore), differences between consecutive $\Delta \varepsilon_{C A M M}^{n}$ surfaces $(n=0 \ldots 9)$ are presented below. Both maximal and average absolute changes are presented, for the entire 100x100 grid used and for a 33x33 central part. All values are given in $\mathrm{kcal} / \mathrm{mol}$.

\begin{tabular}{|c|c|c|c|c|c|c|c|c|}
\hline & \multicolumn{2}{|c|}{$\operatorname{Eth}^{(+1)}-\mathrm{UA} / \mathrm{AU}$} & \multicolumn{2}{|c|}{$\operatorname{Eth}^{(+1)}-\mathrm{GC} / \mathrm{CG}$} & \multicolumn{2}{|c|}{$\mathrm{PF}^{(+1)}-\mathrm{AU} / \mathrm{CG}$} & \multicolumn{2}{|c|}{$\mathrm{PF}^{(0)}-\mathrm{AU} / \mathrm{CG}$} \\
\hline & $100 \times 100$ & $33 \times 33$ & $100 \times 100$ & $33 \times 33$ & 100x100 & $33 \times 33$ & $100 \times 100$ & $33 \times 33$ \\
\hline Maximal change $[\mathrm{kcal} / \mathrm{mol}]$ & & & & & & & & \\
\hline$\Delta \varepsilon_{C A M M}^{0} \rightarrow \Delta \varepsilon_{C A M}^{I^{2}}$ & 7.20 & 7.20 & 6.80 & 6.80 & 5.33 & 4.08 & 6.00 & 6.00 \\
\hline$\Delta \varepsilon_{C A M M}^{I} \rightarrow \Delta \varepsilon_{C A M M}^{I I}$ & 5.46 & 5.26 & 5.60 & 5.60 & 7.36 & 7.36 & 8.38 & 8.38 \\
\hline$\Delta \varepsilon_{C A M M}^{I I} \rightarrow \Delta \varepsilon_{C A M M}^{I I I I}$ & 4.83 & 4.83 & 4.20 & 4.20 & 5.80 & 5.80 & 4.89 & 4.89 \\
\hline$\Delta \varepsilon_{C A M M}^{I I I I I} \rightarrow \Delta \varepsilon_{C A M M}^{I V}$ & 2.27 & 2.27 & 1.94 & 1.94 & 1.79 & 1.79 & 1.99 & 1.99 \\
\hline$\Delta \varepsilon_{C A M M}^{I V} \rightarrow \Delta \varepsilon_{C A M M}^{V}$ & 2.44 & 2.44 & 2.48 & 2.48 & 2.10 & 2.10 & 5.81 & 5.81 \\
\hline$\Delta \varepsilon_{C A M M}^{V} \rightarrow \Delta \varepsilon_{C A M M}^{V I}$ & 0.62 & 0.62 & 0.70 & 0.70 & 0.74 & 0.74 & 2.01 & 2.01 \\
\hline$\Delta \varepsilon_{C A M M}^{V I} \rightarrow \Delta \varepsilon_{C A M M}^{V I I}$ & 0.52 & 0.52 & 0.53 & 0.53 & 0.57 & 0.57 & 0.98 & 0.98 \\
\hline$\Delta \varepsilon_{C A M M}^{V I I} \rightarrow \Delta \varepsilon_{C A M M}^{V I I}$ & 0.43 & 0.43 & 0.33 & 0.33 & 0.29 & 0.29 & 0.47 & 0.47 \\
\hline$\Delta \varepsilon_{C A M M}^{V I I I} \rightarrow \Delta \varepsilon_{C A M M}^{I X}$ & 0.16 & 0.16 & 0.21 & 0.21 & 0.26 & 0.26 & 0.41 & 0.41 \\
\hline Average change $[\mathrm{kcal} / \mathrm{mo}]$ & & & & & & & & \\
\hline$\Delta \varepsilon_{C A M M}^{0} \rightarrow \Delta \varepsilon_{C A M M}^{I}$ & 1.51 & 3.44 & 1.50 & 2.67 & 1.47 & 1.90 & 0.72 & 2.58 \\
\hline$\Delta \varepsilon_{C A M M}^{I} \rightarrow \Delta \varepsilon_{C A M M}^{I I}$ & 0.78 & 2.36 & 0.84 & 2.48 & 0.97 & 2.69 & 0.75 & 3.24 \\
\hline$\Delta \varepsilon_{C A M M}^{I I I} \rightarrow \Delta \varepsilon_{C A M M}^{I I I}$ & 0.37 & 1.41 & 0.36 & 1.49 & 0.47 & 2.20 & 0.39 & 1.57 \\
\hline$\Delta \varepsilon_{C A M M}^{I I I} \rightarrow \Delta \varepsilon_{C A M M}^{I V}$ & 0.23 & 0.84 & 0.21 & 0.66 & 0.21 & 0.77 & 0.23 & 0.87 \\
\hline$\Delta \varepsilon_{C A M M}^{I V} \rightarrow \Delta \varepsilon_{C A M M}^{V}$ & 0.17 & 0.81 & 0.14 & 0.68 & 0.14 & 0.72 & 0.29 & 1.03 \\
\hline$\Delta \varepsilon_{C A M M}^{V} \rightarrow \Delta \varepsilon_{C A M M}^{V I}$ & 0.05 & 0.21 & 0.05 & 0.19 & 0.05 & 0.19 & 0.21 & 0.55 \\
\hline$\Delta \varepsilon_{C A M M}^{V I} \rightarrow \Delta \varepsilon_{C A M M}^{V I I}$ & 0.03 & 0.14 & 0.03 & 0.13 & 0.03 & 0.12 & 0.13 & 0.20 \\
\hline$\Delta \varepsilon_{C A M M}^{V I I} \rightarrow \Delta \varepsilon_{C A M M}^{V I I I}$ & 0.01 & 0.06 & 0.01 & 0.05 & 0.01 & 0.06 & 0.05 & 0.13 \\
\hline$\Delta \varepsilon_{C A M M}^{V I I I} \rightarrow \Delta \varepsilon_{C A M M}^{I X}$ & 0.01 & 0.03 & 0.01 & 0.04 & 0.01 & 0.04 & 0.04 & 0.09 \\
\hline
\end{tabular}

\section{Animation of the studied path on the intercalation plane}

The path connecting the crystallographic position of the ethidium chromophore in the $\operatorname{Eth}^{(+1)}-\mathrm{UA} / \mathrm{AU}$ complex and the minimum in the major groove found on the $\triangle \varepsilon_{C A M M}^{I X}$ surface (Fig.7 in the manuscript) is illustrated by an appended animation. The path is shown as a bold segment in Fig.7. The components of the $\Delta E_{\mathrm{MP} 2}$ interaction energy (Fig.5 and Fig.6 in the manuscript) were studied along this path, which simulates, in a very simplified way, the movement of the intercalator when entering the intercalation site. The animation depicts only the ethidium chromophore, its nearest bases, and the accompanying DNA strands. A transparent van der Waals surface is shown for the DNA fragment to illustrate the tight steric pocket of the intercalation site. The animation was created using the program Visual Molecular Dynamics, version 1.8.2, and mencoder 1.0. 